\title{
Holger Simon
}

\section{Cultural Entrepreneurship - Geschäftsmodelle für Kunst und Kultur}

\section{Einleitung}

Als Larry Page und Sergey Brin im Oktober 2004 persönlich auf der Frankfurter Buchmesse den neuen Dienst Google Prints (heute: Google Books) vorstellten, schreckten sie mit ihrer Idee, alle Bücher im Internet zugänglich zu machen, viele in Europa auf. Verleger, Bibliothekare und Politiker proklamierten gleichermaßen den Ausverkauf der europäischen Kultur. Der Direktor der französischen Nationalbibliothek, Jean Noël Jeanneney, forderte die EU-Staaten in seinem nur wenige Monate später erschienenen Buch "Quand Google défie l'Europe. Plaidoyer pour un sursaut" (Jeanneney 2005) auf, dem Google-Projekt eine europäische Alternative entgegenzusetzen. Die Antwort ließ nicht lange auf sich warten. Am 28. April 2005 schlugen Jacques Chirac und Gerhard Schröder mit weiteren Ministerpräsidenten in einem gemeinsamen Brief den Aufbau einer gemeinsamen digitalen Bibliothek vor, um das kulturelle Erbe Europas in einem europäischen Portal sichtbar zu machen und dies nicht einem amerikanischen Unternehmen zu überlassen.

Damit begann der Wettstreit von zwei Projekten. Beide verfolgen dasselbe Ziel: den Aufbau der größten digitalen Bibliothek mit freiem Zugang für alle. Ihr Geschäftsmodell könnte dagegen kaum unterschiedlicher sein: hier ein non-profit-Unternehmen, welches vom Staat gefördert wird, und dort ein kommerzielles Unternehmen, das Gewinne erwirtschaften will.

Treten hier zwei ungleiche Wettbewerber gegeneinander an? Worin liegen die Chancen und die Gefahren des einen oder anderen Geschäftsmodells? Benötigen wir im Kultursegment andere Geschäftsmodelle als in der Industrie? Welche Auswirkungen hat das Ertragsmodell, also die Frage der Einnahmengenerierung, für das Geschäftsmodell und damit für das operative Geschäft des Projektes? Welche Abhängigkeiten entstehen durch welches Geschäftsmodell?

Jede Organisation hat ein Geschäftsmodell. Um nachhaltig bestehen zu können, muss jede Organisation einen Mehrwert generieren und die anfallenden Kosten durch Einnahmen decken. Damit sind Geschäftsmodelle nicht nur etwas für profitorientierte Unternehmen, sondern auch Kernbestandteil aller non-profit-Organisationen. In der heutigen kulturpolitischen und kulturwirtschaftlichen Diskussion vermisst man das Grundwissen über die Logik von Geschäftsmodellen, wie sie beschrieben und entwickelt werden können. Dies führt dann nicht selten zu polemischen Dichotomien zwischen Kultur und Wirtschaft. Dadurch wird aber das unternehmerische Potenzial von Kultur verschenkt und die nachhaltige Sicherung von kulturellen Angeboten gefährdet. Jeder Kulturschaffende ist aber ein Cultural Entrepreneur, ein Kulturunter- 
nehmer, sobald er ein bestimmtes kulturelles Angebot ermöglichen und erhalten will. Dafür braucht er Methoden, um die erfolgreiche Strategie für das jeweilige Angebot und für die Etablierung der Unternehmung zu finden.

In diesem Artikel soll mit dem Business Model Canvas (BMC) eine Methode vorgestellt werden, mit der wir Geschäftsmodelle exakt beschreiben, lebendig entwickeln und leicht vergleichen können. Jedes Geschäftsmodell gehört stets neu auf den Prüfstand. Anschließend werden vier Kulturportale analysiert und vier Geschäftsmodelle beispielhaft diskutiert.

\section{Business Model Canvas}

Alexander Osterwalder hat im Rahmen seiner Dissertation 2004 bei Yves Pigneur an der HEC Lausanne eine Methode vorgestellt, wie Geschäftsmodellinnovationen entwickelt werden können. Seine Methode wurde schnell zum eigenen Geschäftsmodell. Das 2010 erschienene Buch „Business Model Generation“ ist ein großer Erfolg, nicht nur bei Start-ups, und Alexander Osterwalder ein gut bezahlter Berater im Changemanagement führender Unternehmen. Das Geheimnis hinter seiner Methode ist, dass sie sehr variabel ist, schnell zum Erfolg führt und auch noch Spaß macht (Osterwalder und Pigneur 2011).

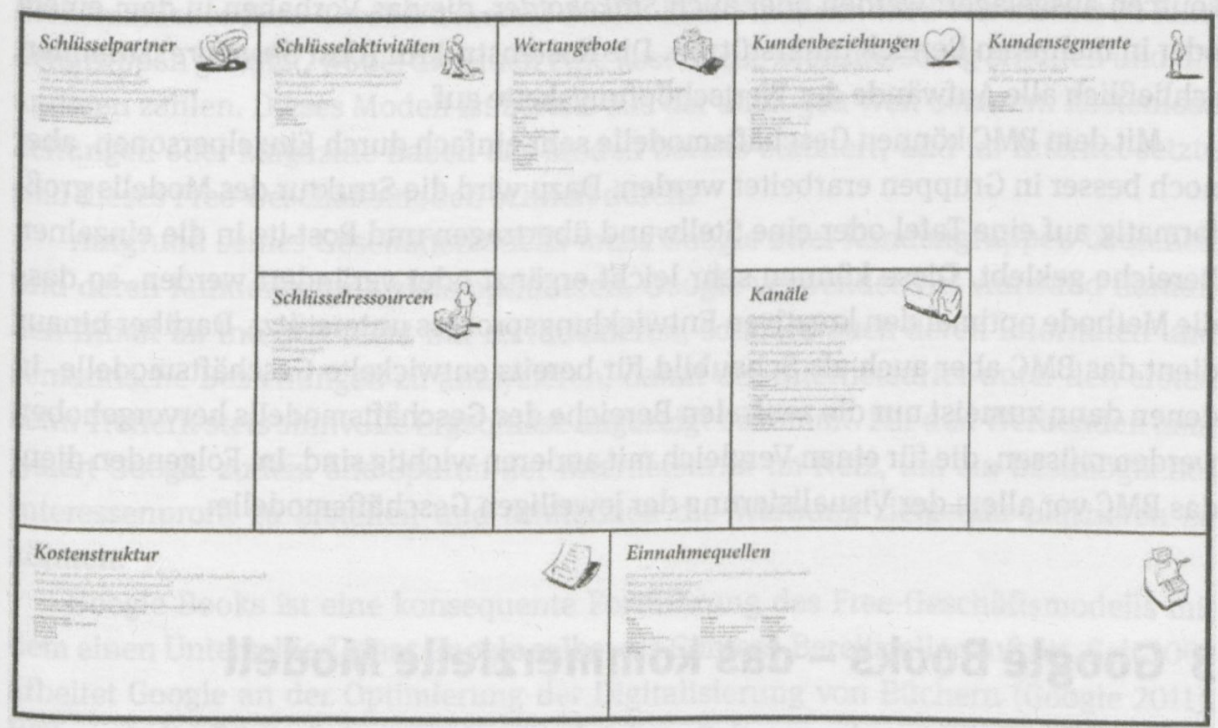

Abb. 1: Business Model Canvas

Das BMC zeigt die zentralen Bausteine eines Geschäftsmodells von der Architektur der Wertschöpfung über das Nutzen- und Wertversprechen bis hin zum Ertragsmo- 
dell. Die neun einzelnen Bausteine stehen in einem ganz bestimmten Verhältnis zu einander.

Im Zentrum des Geschäftsmodells stehen die Wertangebote (Value Propositions) der Produkte und Dienstleistungen, die den Kunden erfolgreich angeboten werden sollen. Hier wird aufgeführt, welches Werteversprechen die Angebote haben und welchen Kundennutzen sie erfüllen. Das Wertangebot teilt das gesamte Geschäftsmodell sodann in zwei Seiten.

Die rechte Seite beschreibt, wie das Wertangebot zum Kunden kommt und wie Erlöse generiert werden. Im Bereich Kundensegmente (Customer Segments) werden die unterschiedlichen Kundengruppen aufgeführt, die eine Organisation bedient. Diese Kunden werden zumeist über unterschiedliche Kanäle (Channels) erreicht. Die Kundenbeziehungen (Customer Relationships) beschreiben, auf welche Weise die Beziehung zu den Kunden gepflegt wird. Einnahmequellen (Revenue Stream) zeigen schließlich die Erlösquellen auf und stellen in der Summe das Ergebnis der erfolgreich angebotenen Produkte und Dienstleistungen dar.

Auf der linken Seite finden wir alle die Elemente der Wertschöpfungskette, die zur Erschaffung des Wertangebots führen und Kosten verursachen. Dies sind zum einen die Schlüsselressourcen (Key Resources), also die Güter oder das Wissen, die für die oben genannten Elemente benötigt werden, und zum anderen die Schlüsselaktivitäten (Key Activities), die das Alltagsgeschäft bestimmen. Die Schlüsselpartner (Key Partnerships) sind entweder die Partner, an die Schlüsselaktivitäten oder -ressourcen ausgelagert werden oder auch Stakeholder, die das Vorhaben in dem einem oder in mehreren Bereich unterstützen. Die Kostenstruktur (Cost Structure) summiert schließlich alle Aufwände der Wertschöpfungskette auf.

Mit dem BMC können Geschäftsmodelle sehr einfach durch Einzelpersonen, aber noch besser in Gruppen erarbeitet werden. Dazu wird die Struktur des Modells großformatig auf eine Tafel oder eine Stellwand übertragen und Post-its in die einzelnen Bereiche geklebt. Diese können sehr leicht ergänzt oder verändert werden, so dass die Methode optimal den kreativen Entwicklungsprozess unterstützt. Darüber hinaus dient das BMC aber auch als Schaubild für bereits entwickelte Geschäftsmodelle, in denen dann zumeist nur die zentralen Bereiche des Geschäftsmodells hervorgehoben werden müssen, die für einen Vergleich mit anderen wichtig sind. Im Folgenden dient das BMC vor allem der Visualisierung der jeweiligen Geschäftsmodelle.

\section{Google Books - das kommerzielle Modell}

Kehren wir also zurück zum Ausgangspunkt und den beiden Projekten, die beide eine digitale Bibliothek anbieten, deren Geschäftsmodell sich aber stark unterscheidet. Zum Verständnis der strategischen Funktion von Google Books lohnt ein kurzer Blick in die Anfänge von Google. 
Google hat seinen Ursprung in der Buchsuche. Larry Page und Sergey Brin entwickelten an der Stanford University in Kalifornien einen Webcrawler, um die zunehmend digitalisierten Bücher im Internet in einer großen Bibliothek recherchierbar und zugänglich zu machen. So lag es auf der Hand, dass sie sich wenige Jahre nach Gründung des Unternehmens wieder der digitalen Bibliothek zuwendeten, zumal diese ihr Kerngeschäft, die Suchmaschine, direkt ergänzt.

Das Bestreben von Google ist, „die Informationen der Welt zu organisieren und für alle zu jeder Zeit zugänglich und nutzbar zu machen“ (Google 2015). Das zentrale Wertangebot von Google ist damit klar: Informationen schnell finden. Damit trifft Google ein Grundbedürfnis aller Nutzer im Internet. Um möglichst viele Nutzer an sich zu binden, ist das Angebot von Google sehr niederschwellig: Es ist kostenlos und mit einem Suchschlitz sehr einfach handhabbar. Um dieses Wertangebot zu ermöglichen, benötigt Google Spezialisten für die Entwicklung und ständige Anpassung des Suchalgorithmus, mächtige Server für die Indizierung und eine Suchplattform. Finanziert wird diese kostenlose Dienstleistung durch Werbung. Seit 2000 bietet Google den Werbedienst AdWords an, mit dem Werbende über Schlüsselbegriff-Auktionen bei ihren Kunden sehr zielgerichtet Werbung platzieren können. Um ihren eigenen Umsatz durch Werbeeinnahmen noch weiter zu erhöhen, stellt Google außerdem das System AdSense zur Verfügung, mit dem Betreiber von Webseiten auf ihren eigenen Portalen Google-Werbung schalten und so selber Einnahmen erzielen können.

Mit der Suchmaschine setzt Google sehr konsequent eine Multi-sided Platform als herkömmliches Free-Geschäftsmodell durch. Eine Plattform wird von mehreren Kundengruppen genutzt, wobei die einen eine kostenlose Dienstleistung erhalten und die anderen zahlen. Dieses Modell ist bereits aus der analogen Welt bekannt. Kostenlose Zeitungen oder Magazine haben das Modell bereits etabliert, und im Internet setzte sich dieses Free-Geschäftsmodell schnell durch.

Aufgrund seines Geschäftsmodells muss Google zwei Kundengruppen bedienen und deren Kundennutzen stets optimieren. Google verwendet viel Aufwand darauf, den Inhalt im Internet nicht nur zu indexieren, sondern auch deren Prioritäten und semantische Beziehungen zu analysieren, damit der Internetsurfer unter den ersten zehn Treffern stets sinnvolle Ergebnisse angezeigt bekommt. Für den Werbenden analysiert Google zudem alle Spuren der Internetsurfer im Netz, um ein bestmögliches Interessenprofil $\mathrm{zu}$ erstellen und schließlich die Werbung zielgenau platzieren $\mathrm{zu}$ können.

Google Books ist eine konsequente Fortführung des Free-Geschäftsmodells mit dem einen Unterschied, dass Google selbst als Content-Bereitsteller auftritt. Seit 2002 arbeitet Google an der Optimierung der Digitalisierung von Büchern (Google 2011). Während die Bibliotheken noch zur gleichen Zeit von einem Jahrhundertprojekt sprachen, schlug Google den Bibliotheken vor, die Bücher in wenigen Jahren zu digitalisieren. Dass auch dieses Angebot wiederum kostenlos den Bibliotheken angeboten wurde, liegt auf der Hand und folgt der Logik des Geschäftsmodells. Je mehr hochwertiger Content im Internet zur Verfügung steht, desto zielgerichteter kann 
Google bei der Suche seine Werbung platzieren. Die Aufwendungen der Digitalisierung von einigen 100 Mio. Dollar erscheinen gegenüber den Milliardengewinnen durch Werbung als zweckdienliche Investitionskosten.

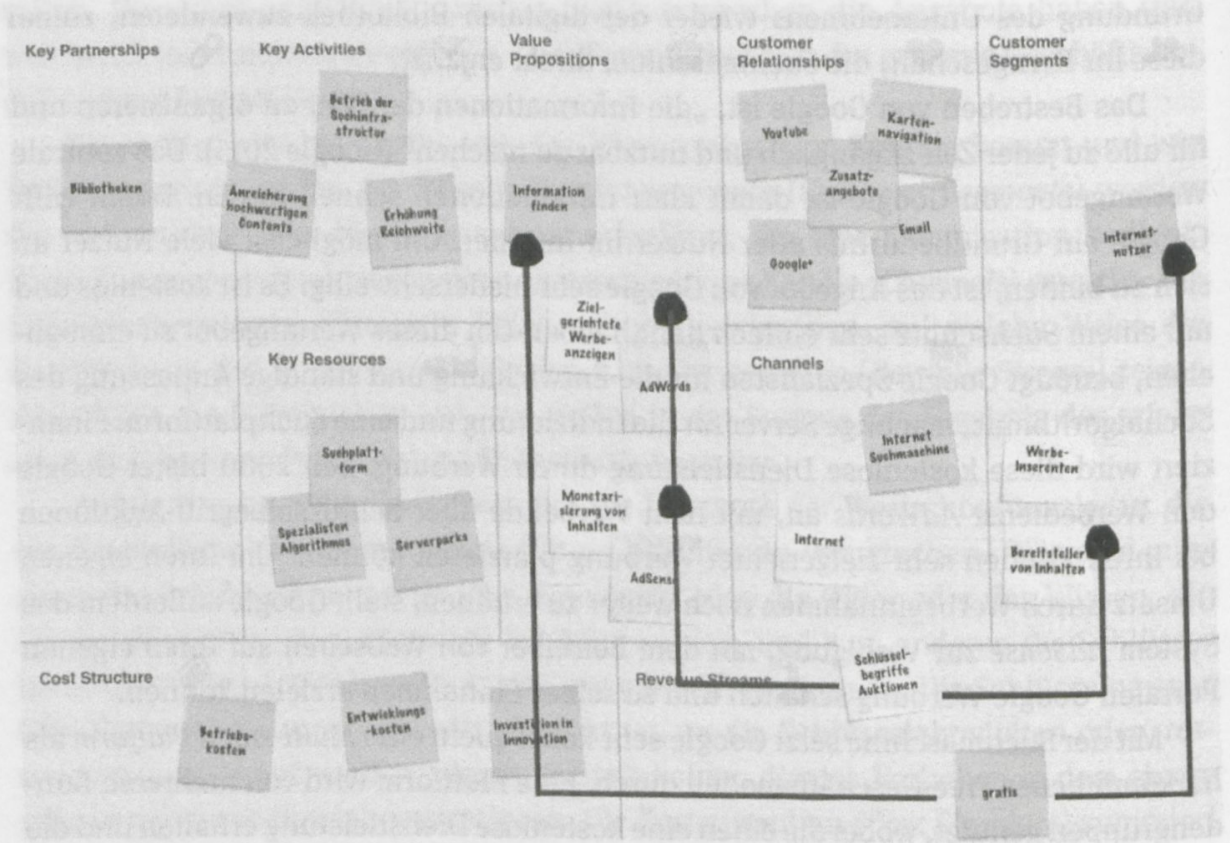

Abb. 2: BMC für Google

Den von Verlagen und Autoren ausgelösten jahrelangen Rechtsstreit in den USA konnte Google für sich entscheiden, da das Unternehmen sich bei der Digitalisierung der Bücher in den USA auf die Doktrin des Fair Use bezog. Die Geschworenen sprachen Google nicht nur von den Vorwürfen der Urheberrechtsverletzung frei, sondern Richter Chin setzte auch noch einen drauf, indem er Google Books lobte, weil es „vergessene Werke rettet und ihnen neues Leben einhaucht“ sowie darüber hinaus „neues Publikum und neue Einnahmen“ schafft (Sokolov 2013). In Europa hingegen werden urheberrechtlich geschützte Werke nur in wenigen Auszügen angezeigt und nicht vollständig zugänglich gemacht.

Der Vorteil dieses Geschäftsmodells ist, dass Google mit der Multi-sided Platform die Bedürfnisse seiner beiden Kundengruppen, Internetnutzer und Werbende, sehr klar ermitteln kann. Beide Gruppen brauchen sich notwendigerweise gegenseitig, da die eine das Angebot kostenlos nutzen und die andere ihre Kunden zielgerichtet mit Werbung erreichen möchte. Damit hat Google ein sehr stabiles Geschäftsmodell, das nur durch Wettbewerber gefährdet werden kann, die beiden Gruppen einen besseren Mehrwert anbieten können. Eingedenk dessen versucht Google, seine kostenlosen Angebote auszuweiten, um seine Kunden dauerhaft an sich zu binden, z. B. durch 
Google Maps, Gmail, YouTube, Google Docs oder das Betriebssystem Android. Denn auch hier entstehen wichtige Daten für den mächtigen Google-Algorithmus. Der bedeutende Content in dem Portal Google Books schließt hier nahtlos an.

Das Geschäftsmodell birgt aber auch Gefahren, die vor allem dadurch entstehen, dass Google ein enormes Wissen über die persönlichen Profile seiner Nutzer generiert, die nicht in falsche Hände kommen dürfen. Daran kann das Unternehmen kein Interesse haben. Denn seine Nutzer sind sein höchstes Gut, ohne sie brechen die Werbeeinnahmen schlagartig weg, z. B. wenn der Nutzer seine Daten bei Google nicht in sicheren Händen weiß. Ein Angriff auf die Schlüsselressourcen, in dem Staaten wie die USA in gemeinen Gerichtsurteilen Firmen wie Google, Facebook und Apple dazu zwingen, ihre Daten zugänglich zu machen (Fokus 2014), ist ein Angriff auf die Persönlichkeitsschutzrechte der Nutzer. Wohl gemerkt von den Staaten und nicht von den Unternehmen. Das gleiche gilt für Staaten wie Deutschland, die befreundeten Nachrichtendienste ihre Zugänge für Spionage freigeben.

\section{Europeana - Das Fördermodell}

Diese Gefahr der Spionage muss der Antipode von Google Books, die Europeana, nicht befürchten. Zum einen werden die Daten der Europeana bereits von staatlichen Einrichtungen selbst bzw. in deren Auftrag gehostet und zum anderen ist es nicht Teil des Geschäftsmodells, Werbung zielgerichtet zu platzieren, wofür ein detailliertes Wissen über die Vorlieben der Nutzer notwendig ist. Aber auch die Europeana hat wie Google zwei Kundengruppen, eine, die kostenlos einen Dienst nutzt und eine, die zahlt. Im Unterschied zu Google sind diese Kundengruppen bei der Europeana aber nicht über eine Multi-sided Platform abhängig aneinander gebunden. Vielmehr erhält der eine einen Dienst, für den der andere zahlt.

Das zentrale Wertangebot der Europeana ist, das europäische Kulturerbe digital über das Portal zugänglich zu machen. Die Europeana konzentriert sich dabei nicht nur auf Bücher, sondern möchte alle Objekte aus Museen, Archiven und Bibliotheken digital zugänglich machen. Kunden sind demnach alle, die Quellen und digitale Objekte des kulturellen Erbes suchen. Hier hat die Europeana neben Wissenschaft und Bildung auch die Wirtschaft und vor allem die Tourismusindustrie im Blick, die durch die freie und kostenlose Verwendung der Inhalte kommerzielle Angebote entwickeln soll.

Neben diesen Internetnutzern hat die Europeana aber noch einen weiteren Kunden. Die Kosten von zur Zeit bis zu 5 Millionen $€ / J a h r$ werden fast vollständig von der Europäischen Kommission getragen. Die Europäische Kommission wird damit zum Kunden, weil sie die Einnahmen aufgrund eines Wertversprechens sicherstellt. Dieses Wertversprechen war zu Beginn stark getrieben von zwei Motiven: Google 
Books eine europäische digitale Bibliothek entgegenzusetzen und das kulturelle Erbe Europas digital zugänglich zu machen.

Damit das Fortbestehen der Europana auch zukünftig sichergestellt werden kann, muss die Kommission als Kunde besonders bedient werden. Und das hat Auswirkungen auf die Schlüsselaktivitäten des Geschäftsmodells. Denn die Europeana muss bei jeder Vertragsverlängerung die Kommission bzw. die EU-Staaten neu davon überzeugen, dass das Fortbestehen des Portals von besonderer Wichtigkeit ist. Hierzu ist viel Lobbyarbeit nötig. Der Strategieplan 2011-2015 war ein wichtiger und notwendiger Schritt für eine innovative Idee und war notwendig nach einem verstolperten Start. Der Strategieplan dient aber vor allem dazu, dem zentralen Geldgeber einen Weg aufzuzeigen, wohin die Europeana strebt, und für ihn Kundennutzen zu generieren. Das Paper bietet deswegen viele Argumentationshilfen für die Abgeordneten und zeigt mit den Schlüsselaktivitäten „Sammeln“, „Fördern“, „Verbreiten“ und „Beteiligen“ sichtbare und politisch gewollte Aktivitäten. Schließlich wird in dem Strategieplan sehr differenziert der externe, direkte und indirekte Nutzen des Portals für Europa vorgelegt.

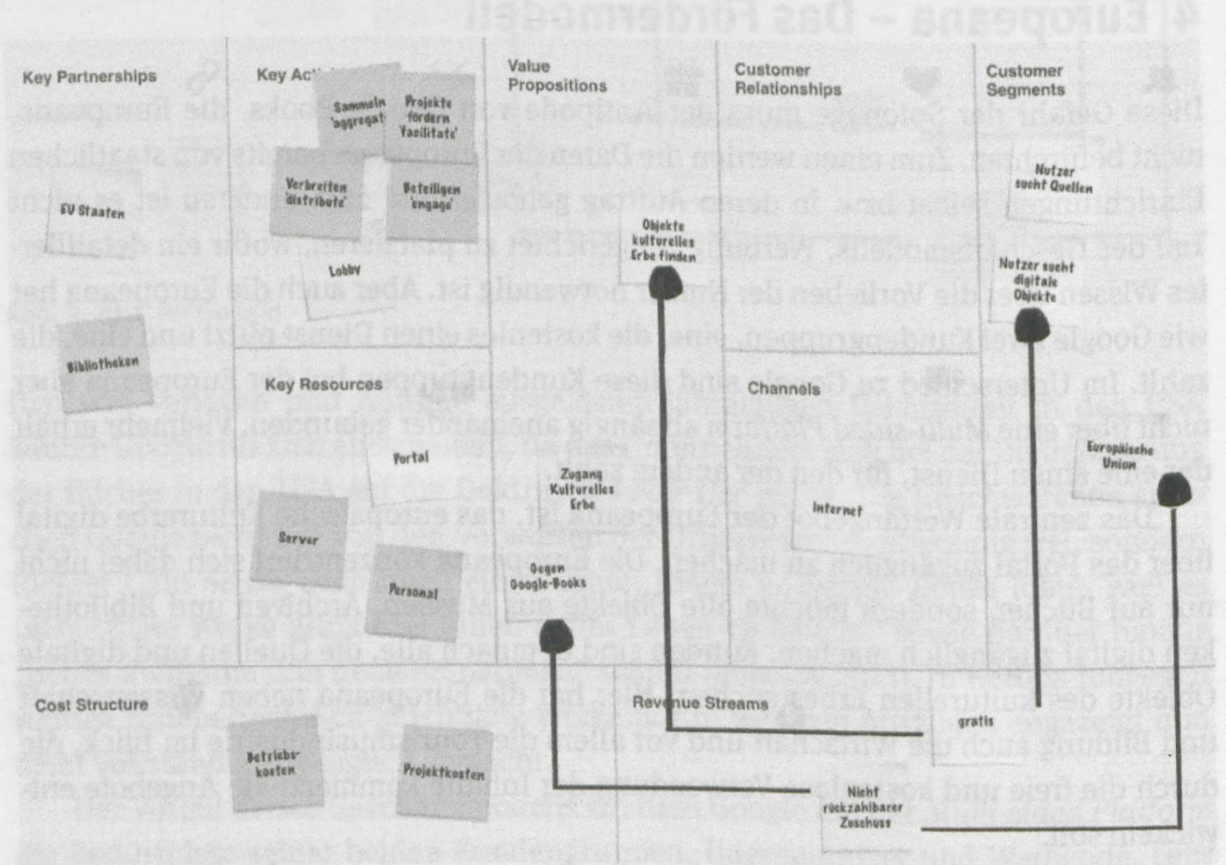

Abb. 3: BMC für die Europeana

An dieser Stelle gleicht das Geschäftsmodell vielen weiteren öffentlich finanzierten Projekten und damit einem Großteil der im Folgenden vorgestellten Kulturportale. Die Stärke dieses Geschäftsmodells ist, dass das Portal nicht von einem Endkunden abhängig ist, der für die Dienstleistung direkt oder indirekt bezahlen soll. Außerdem 
ist die Anzahl der Nutzer nicht von relevantem wirtschaftlichem Interesse, denn die eigentliche Herausforderung besteht darin, die öffentliche Förderquelle nicht versiegen zu lassen. Viele von diesen Projekten haben daher nur eine begrenzte Laufzeit oder nehmen die öffentliche Hand anschließend zunehmend in die Pflicht.

Auf der anderen Seite erfordert dieses Geschäftsmodell von den Akteuren, den Blick auf den eigentlichen Endverbraucher und die Generierung seines Kundennutzens nicht zu verlieren, für den die Anwendung einst geschaffen wurde. Daher will die Europeana die Nutzer aktiv einbinden und beteiligen.

Ähnlich wie bei Google kann in der Europeana nur das recherchiert werden, was die Museen, Archive und Bibliotheken dem Portal zur Verfügung stellen. Sie sind in dem Geschäftsmodell Schlüsselpartner, da sie innerhalb der Wertschöpfungskette einen zentralen Beitrag, nämlich den Content liefern. Sie könnte man - ähnlich wie beim Bildarchiv prometheus (siehe unten) - aber auch als Kunden anführen. Da sie aber von der Europeana selber nicht aktiv akquiriert werden, sondern dies in die Verantwortung der einzelnen EU-Länder übergeben wurde, verstehen wir sie hier als Schlüsselpartner. Eine Herausforderung besteht hierbei darin, die Daten so geliefert zu bekommen, dass die Endverbraucher die Inhalte verwenden können und davon einen Mehrwert haben. Dies ist ein neuralgischer Punkt, denn es fällt der Europeana schwer, eine Creative-Commons-Lizenz Null (CCO) und eine hohe Auflösung für ihre Objekte durchzusetzen. Infolgedessen beschränken sich bisher die Anwendungen der Tourismusindustrie ebenso wie die der Wissenschaft auf nur wenige beispielhafte Projekte, die wenig Mehrwert bieten. Darüber hinaus wird die Digitalisierung der Museen, Archive und Bibliotheken noch viele Aufwände erzeugen und Zeit kosten, bis wirklich nennenswerte Mehrwertanwendungen geschaffen werden können.

Google hat diesen Bedarf natürlich erkannt und nutzt auch dies zu seinem Vorteil. Da nun der Staat von den Kultureinrichtungen die Digitalisierung einfordert, aber zugleich längst nicht genügend Mittel zur Verfügung stellt, springt Google ein und hilft. Der Suchmaschinenriese kommt wie ein Wohltäter daher, nutzt aber vor allem die Chance, die Digitalisierung zu beschleunigen und die Inhalte für den eigenen Gebrauch zu analysieren, um seine Werbung in der Suchmaschine besser zu platzieren.

Dieses Dilemma kann man in diesem Geschäftsmodell nur lösen, indem der zahlende Kunde - hier der Staat - viel mehr Gelder für die Digitalisierung bereitstellt, oder aber der Mehrwert für den Endkunden muss derart gesteigert und viele Nutzer gebunden werden, damit über sie z. B. durch Crowdfunding oder einem jährlichen Spendenaufruf die notwendigen Mittel eingenommen werden können. Wikipedia setzt ein solches Geschäftsmodell seit ihrer Gründung sehr erfolgreich um. Bisher machen die Nutzerzahlen der Europeana ein solches Modell allerdings unwahrscheinlich. 


\section{5 prometheus-Bildarchiv - Das non-profit-Modell}

In den Jahren 2001 bis 2004 wurde vom Bundesministerium für Bildung und Forschung das prometheus-Bildarchiv mit dem Ziel gefördert, ein verteiltes Bildarchiv für die Kunstgeschichte und Archäologie zu entwickeln. Mit Auslaufen der Förderung stand prometheus kurz vor dem Projekt-Aus. Über 30 Institute nutzten aber bereits das Archiv für die tägliche Arbeit, und viele Institute hatten schon begonnen ihre Diatheken abzubauen. Folglich war der Druck groß, eine Finanzierungslösung zu finden und ein nachhaltiges Geschäftsmodell für prometheus zu entwickeln.

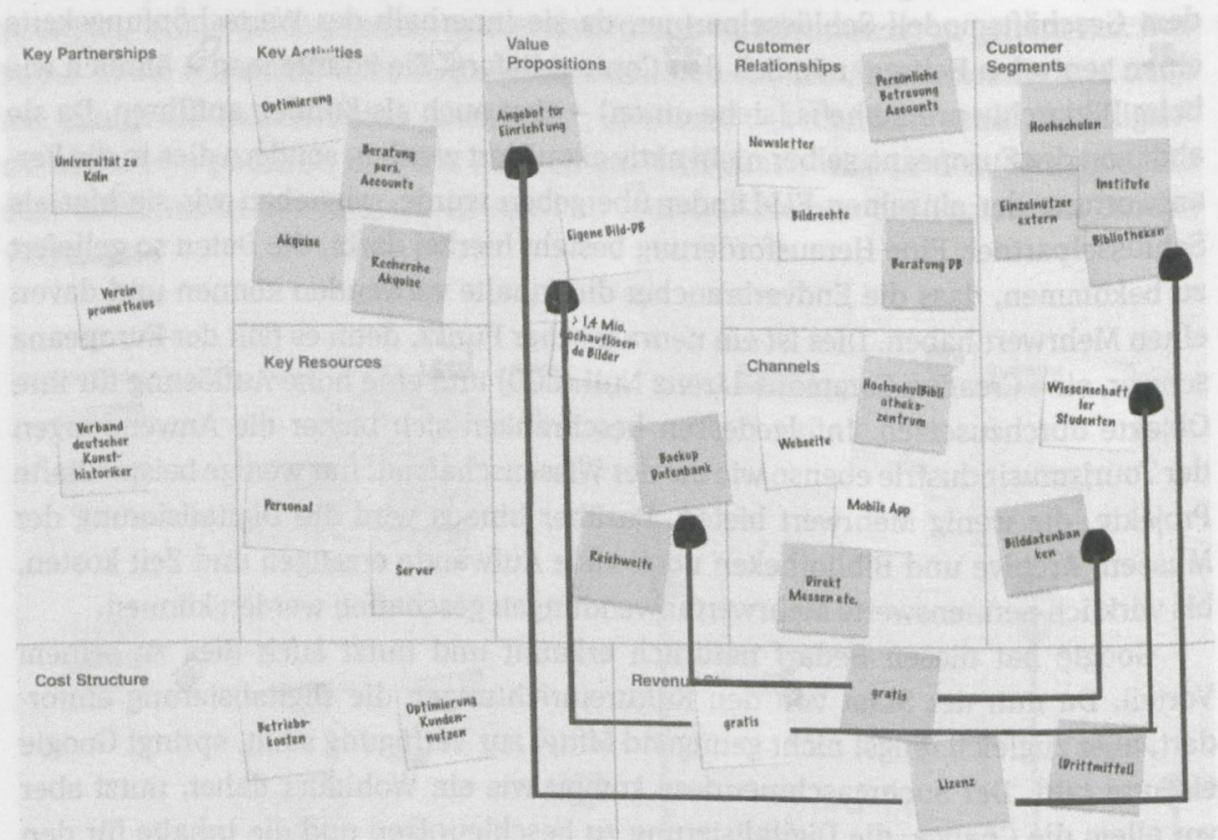

Abb. 4: BMC für das prometheus-Bildarchiv

prometheus hat eine zentrale Nutzergruppe: Wissenschaftler und Studierende, vor allem der Fächer Kunstgeschichte und Archäologie, mit heute durchschnittlich über 15.000 Suchanfragen pro Tag. Die Nutzer finden hier in den über 1,3 Millionen hochauflösenden Bilder einen Großteil ihrer Studienobjekte (Stand: Mai 2015). Fehlen Bilder, kann bei den meisten Instituten die Digitalisierung beantragt werden, die anschließend prometheus zur Verfügung gestellt werden. Zudem können eigene Bilder direkt hochgeladen werden. So wird das notwendige Bildrepertoire zum Vorteil aller Kunden stetig erweitert. Die Bilder können über die Website von prometheus oder die eigene Smartphone-App gesucht und für Präsentationen und Forschungsarbeiten verwendet werden. Diese Dienstleistung ist für die Wissenschaftler und Studierenden gratis. 
Ähnlich wie bei der Europeana wird die Qualität des Portals durch die Qualität der Objekte bestimmt. Die integrierten Bilddatenbanken der Institute, Bibliotheken und Museen müssen alle persönlich akquiriert werden, so dass sie in diesem Geschäftsmodell auch Kunden sind. Sie erhalten als Wertversprechen Reichweite für ihre Bilder und eine Webpräsenz für die eigenen Studierenden, weil die Datenbanken lokal vor Ort für die Studierenden meist nicht nutzbar sind. Für einige Datenbanken dient prometheus sogar als externes Backup. Und Forschungsdatenbanken nehmen gerne den Service wahr, dass ihre Daten nach Projektende bei prometheus gehostet werden und damit der Forschung langfristig zugänglich bleiben.

Die zahlenden Kunden sind vor allem die Hochschulen, Bibliotheken und Institute. Sie zahlen eine jährliche Lizenz und erhalten damit Zugänge für ihre lokalen Rechner und einen Admin-Account, mit dem sie persönliche Zugänge für Wissenschaftler und Studierende kostenlos vergeben können. Das Wertversprechen für diese Institutionen ist ein digitales Angebot, das deren Mitglieder benötigen. Einzelnutzer, die keiner Institution angehören, können durch eine geringe Einzellizenz das Bildarchiv ebenfalls nutzen.

Schlüsselpartner ist ein gemeinnütziger Verein, der nach dem Projektende die Rechte von allen neun Projektpartnern gebündelt und den Geschäftsbetrieb an die Universität zu Köln abgegeben hat. Die Dienstleistung als solche wurde als eine hoheitliche Aufgabe anerkannt und ist somit von der Mehrwertsteuer befreit. Dadurch dürfen aber auch keine Gewinne gemacht und die eingenommenen Gelder müssen für diese Tätigkeit ausgegeben werden.

Dieses non-profit-Modell wurde 2005 eingeführt und es trägt seit $2008 \mathrm{zu} 100 \%$ die Betriebs- und Weiterentwicklungskosten des Bildarchivs. Uber Drittmittelprojekte werden Innovationen in den Bereichen Suche, Indexierung und Performance und Kundenanwendungen vorangetrieben.

Die Stärke des Geschäftsmodells liegt klar in der Unabhängigkeit von einer öffentlichen Förderung. Nur wenn viele Nutzer der jeweiligen Hochschule das Bildarchiv täglich nutzen, sind die Hochschulen auch bereit, die Lizenz zu zahlen. Die Schlüsselaktivitäten beziehen sich daher vor allem auf den Ausbau des Kundennutzens, indem die Suchfunktionen weiter optimiert und die Anzahl hochauflösender Bilder erweitert wird. Die Hochschulen und Institute werden bei der Digitalisierung vor Ort unterstützt und beraten.

Die Schwierigkeiten dieses Geschäftsmodells liegen in der Skalierung. Die zahlenden Kunden sind im deutschsprachigen Raum endlich, so dass prometheus mit der Kundenakquise im europäischen und angloamerikanischen Raum begonnen hat. Dies erfordert aber Investitionen in Mehrsprachigkeit und Vertriebsstrukturen, die ein solches non-profit-Geschäftsmodell aus dem Cashflow bezahlen muss, weil Investoren mit Hoffnung auf Rendite nicht gewonnen werden können. Eine engere Zusammenarbeit mit der Europeana könnte an dieser Stelle für beide Seiten nützlich sein. 


\section{6 digiCult - Das gemischte Modell}

Das Museumsportal digiCult ging ebenfalls aus einem geförderten Projekt hervor. Zur langfristigen Sicherstellung des Portals wurde ein gemischtes Geschäftsmodell gewählt, das eine für Kulturportale sehr interessante Variante aufzeigt.

Der Endkunde ist der Internetsurfer, für den der Zugriff auf dem Portal kostenlos ist. Ihr Wertangebot ist der Zugang zu digitalen Objekten aus den Museen des Verbundes.

Zur Finanzierung des Projektes wurde ein kommerzieller Weg gewählt, gekoppelt an eine finanzielle Förderung durch das Land. Dafür wurde als Rechtsform eine eingetragene Genossenschaft $(\mathrm{e} G)$ gegründet. Jedes Verbundmitglied, das seine Daten über das Portal veröffentlichen und die Vorteile nutzen will, muss je nach Größe einen Genossenschaftsanteil kaufen und zugleich einen jährlichen Beitrag je nach Rechtsform und Anzahl der Vollzeit-Mitarbeiter zahlen. Von den 300.000 EUR, die zum Betrieb und Fortführung des Museumsportals benötigt werden, können die Verbundmitglieder so mindestens $1 / 5$ selber aufbringen. Die restlichen $4 / 5$ werden dann über Länderbeiträge sichergestellt.

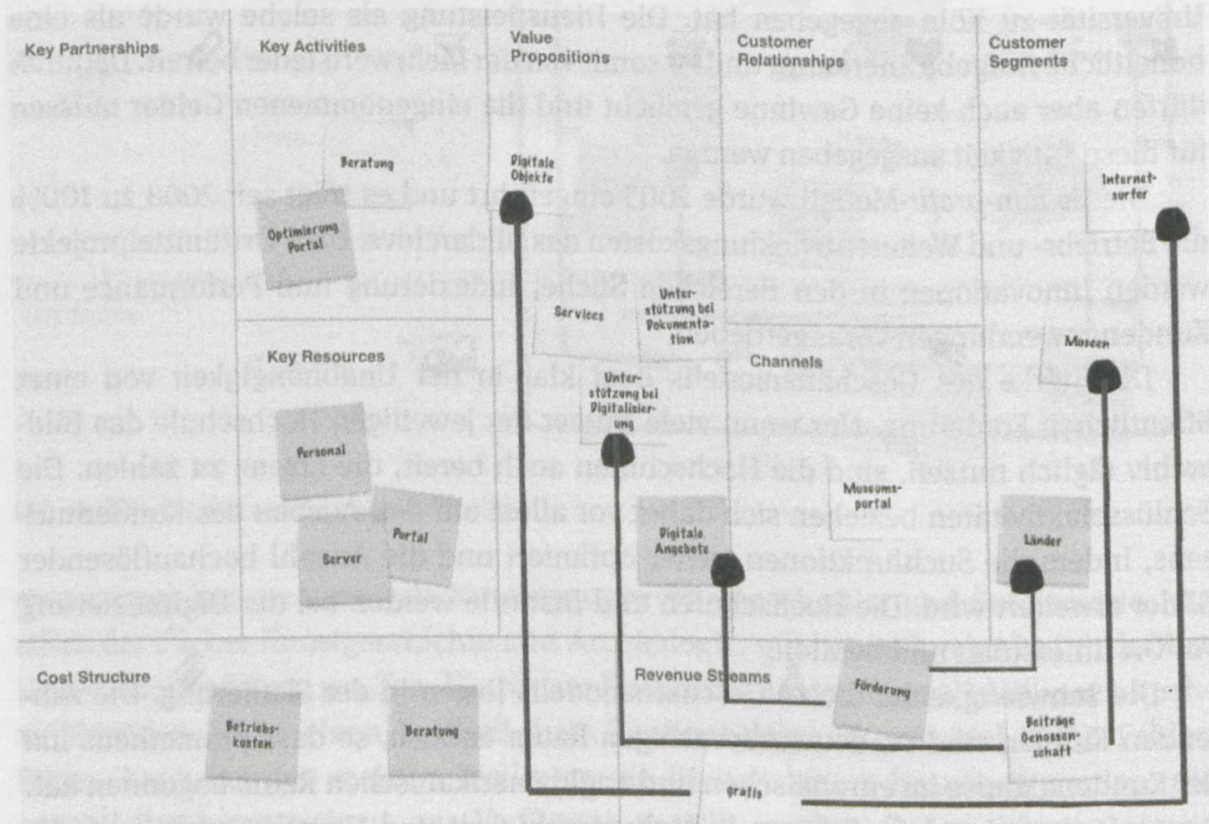

Abb. 5: BMC für digicult

Dieses Geschäftsmodell hat seinen besonderen Charme, weil es zum einen die Nachfrage der Digitalisierung und Zurverfügungstellung der digitalen Objekte in einer Genossenschaft bündelt und zum anderen kommerzielle Tätigkeit ermöglicht. Mögli- 
che Gewinne verbleiben im Verbund, über dessen Verwendung die Verbundmitglieder selber bestimmen. Durch die Bündelung der Nachfrage und die Eigenbeteiligung fällt es den Ländern wiederum leichter, den Verbund zu bezuschussen, um den Erhalt und Ausbau des Museumsportals zu gewährleisten.

Durch das Genossenschaftsmodell kommt natürlich eine neue Herausforderung ins Unternehmen: Die Meinungen der Verbundmitglieder müssen kanalisiert und deren Beratungs- und Unterstützungsbedarf durch unterschiedliche Services stets befriedigt werden. Der größte Mehrwert dieses Modells liegt sicherlich in der gegenseitigen Stärkung auf dem Wege der Digitalisierung.

Eine weitere Herausforderung liegt bei diesem Geschäftsmodell, ähnlich wie beim Fördermodell der Europeana, darin, dass der eigentliche Nutzer und seine Bedürfnisse hinter die Bedürfnisse der Verbundmitglieder treten. Denn für den Erhalt des Verbundes, der vor allem ein Distributionsverbund ist, ist es nicht relevant, ob das Portal auch wirklich nennenswert genutzt wird. Natürlich muss dies nicht das Ziel des Projektes sein, denn auch eine Bibliothek bewahrt ebenfalls Bücher auf, die sehr selten gelesen werden. Es ist aber wichtig für die Strategie und Kommunikation des Verbundes nach außen und für die Herausarbeitung der unterschiedlichen Intentionen, die durch Geschäftsmodelle bestimmt werden. Denn die Folge sind nicht selten nahezu unbenutzte Webseiten.

Eine große Chance besteht für ein solches gemischtes Geschäftsmodell darin, dass der Verbund kommerziell tätig sein kann und seine besonderen Fähigkeiten in Technologie und Beratung an andere Verbünde und Museen kostenpflichtig anbieten und monetisieren könnte. Die Genossenschaft kann als Wirtschaftsbetrieb weitere Erlösquellen etablieren und somit die Einnahmequellen schrittweise ausbauen, die letztendlich wiederum dem Erhalt des Verbundes zu Gute kommen.

\section{Cultural Entrepreneurship}

Die vier verschiedenen Geschäftsmodelle, die hier vorgestellt wurden, weisen jeweils unterschiedliche Chancen und Herausforderungen auf. Die Methode des BMC ist nicht nur ein gutes Hilfsmittel zur Visualisierung dieser Geschäftsmodelle, sondern auch für die Entwicklung und den Ausbau von unternehmerischen Strategien nützlich. Vor allem können mit dem BMC die Intentionen eines Geschäftsmodells über das Wertversprechen der einzelnen Kunden analysiert werden, die direkten Einfluss auf die Schlüsselaktivitäten und damit das operative Geschäft haben. Kunst und Kultur brauchen differenzierte Geschäftsmodelle, die auf ihren besonderen Zweck zugeschnitten sind und kulturelle Angebote langfristig sichern wollen.

Der Cultural Entrepreneur ist in Anlehnung an Bygrave und Hofer ein Unternehmer, der Chancen in der Kultur erkennt und die Ressourcen und Organisation dafür schafft, diese Chancen zu realisieren (Halberstadt und Simon 2014). Das Wissen 
über Geschäftsmodelle und ihre Gestaltungsmöglichkeit ist dafür notwendig und ein Gewinn für die Kultur. Denn die Rendite des Cultural Entrepreneurs ist nicht nur der finanzielle Gewinn - den braucht er zwar auch, um sein Kulturangebot auszubauen und zu investieren -, vielmehr liegt sein Gewinn aber in der Sicherstellung und nachhaltige Ermöglichung von Kulturangeboten. Eingedenk dessen brauchen wir mehr Kulturunternehmer.

\section{Literatur}

Bygrave, W. D. und C. W. Hofer. Entrepreneurship. Theory and Practice, 1991.

Europeana Strategic Plan 2011-2015. http://pro.europeana.eu/files/Europeana_Professional/ Publications/Strategic\%20Plan\%202011-2015\%20-\%20DE.pdf (12. Mai 2015).

Fokus. US-Regierung drohte Yahoo mit Millionenstrafe, Fokus 12. September 2014. http://www. focus.de/finanzen/news/nsa-affaere-yahoo-sollte-250-000-dollar-strafe-zahlen_id_4127515. html (12. Mai 2015).

Die Geschichte der Google Buchsuche. http://www.google.de/intl/de/googlebooks/history.html (12. Mai 2015).

Halberstadt, Jantje und Holger Simon. Cultural Entrepreneurship als Herausforderung - Wie können kulturelle Aufgaben und Angebote unternehmerisch wirksam unterstützt werden? Memorandum der 49. Sylter Runde vom 10./11. April 2014. http://www.sylter-runde.de/mediapool/6/63715/ data/SR_49_CulturaL_Entrepreneurship_download.pdf http://www.focus.de/finanzen/news/ nsa-affaere-yahoo-sollte-250-000-dollar-strafe-zahlen_id_4127515.html (12. Mai 2015).

Jeanneney, Jean Noël. Quand Google défie l'Europe. Plaidoyer pour un sursaut, Editions Mille et une nuits. Paris, 2005. Dt. Fassung: Jean-Noël Jeanneney. Googles Herausforderung. Für eine europäische Bibliothek. Aus dem Französischen von Nathalie Mälzer-Semlinger und Sonja Fink. Berlin, 2006.

Lischka, Konrad. „Digitale Bibliotheken: Der Staat spart, Google digitalisiert“. Spiegel online, 26. März 2011, http://www.spiegel.de/netzwelt/netzpolitik/digitale-bibliotheken-der-staatspart-google-digitalisiert-a-753229.html (12. Mai 2015).

Osterwalder, Alex und Yves Pigneur. Business Model Generation. Ein Handbuch für Visionäre, Spielveränderer und Herausforderer, Frankfurt am Main, 2011.

Sokolov, Daniel A. J. Hintergrund: Warum Google Books in den USA legal ist. 15. November 2013. http://www.heise.de/newsticker/meldung/Hintergrund-Warum-Google-Books-in-den-USAlegal-ist-2046820.html (12. Mai 2015).

Über Google. https://www.google.de/intl/de/about/ (12. Mai 2015). 